\title{
The Role of the Respiratory Microbiome and Viral Presence in Lower Respiratory Tract Infection Severity in the First Five Years of Life
}

\author{
Ivo Hoefnagels ${ }^{1}$, Josephine van de Maat ${ }^{1,2}$, Jeroen J.A. van Kampen ${ }^{3}{ }^{\circ}$, Annemarie van Rossum ${ }^{4}$, \\ Charlie Obihara ${ }^{5}$, Gerdien A. Tramper-Stranders ${ }^{6}$, Astrid P. Heikema $\left.{ }^{7}{ }^{(}\right)$, Willem de Koning ${ }^{8}{ }^{(D}$, \\ Anne-Marie van Wermerskerken ${ }^{9}$, Deborah Horst-Kreft ${ }^{7}$, Gertjan J.A. Driessen ${ }^{10}$, Janine Punt ${ }^{11}$, Frank J. Smit ${ }^{12}$, \\ Andrew Stubbs ${ }^{8}$, Jeroen G. Noordzij ${ }^{13}$, John P. Hays ${ }^{7}\left[\right.$ and Rianne Oostenbrink ${ }^{1, * \mathbb{C}}$
}

check for updates

Citation: Hoefnagels, I.; van de Maat, J.; van Kampen, J.J.A.; van Rossum, A.; Obihara, C.; Tramper-Stranders, G.A.; Heikema, A.P.; de Koning, W.; van Wermerskerken, A.-M.; Horst-Kreft, D.; et al. The Role of the Respiratory Microbiome and Viral Presence in Lower Respiratory Tract Infection Severity in the First Five Years of Life. Microorganisms 2021, 9 , 1446. https://doi.org/10.3390/ microorganisms 9071446

Academic Editor: Tjip S. van der Werf

Received: 31 May 2021

Accepted: 26 June 2021

Published: 5 July 2021

Publisher's Note: MDPI stays neutral with regard to jurisdictional claims in published maps and institutional affiliations.

Copyright: (C) 2021 by the authors Licensee MDPI, Basel, Switzerland. This article is an open access article distributed under the terms and conditions of the Creative Commons Attribution (CC BY) license (https:/ / creativecommons.org/licenses/by/ $4.0 /)$.
1 Department of General Pediatrics, Erasmus MC-Sophia Children's Hospital, 3015GD Rotterdam, The Netherlands; i.hoefnagels@erasmusmc.nl (I.H.); Josephine.vandeMaat@radboudumc.nl (J.v.d.M.)

2 Department of Internal Medicine, Radboud Center of Infectious Diseases, Radboudumc, 6525GA Nijmegen, The Netherlands

3 Department of Viroscience, Erasmus University Medical Centre (Erasmus MC), 3015GD Rotterdam, The Netherlands; j.vankampen@erasmusmc.nl

4 Departement of Pediatrics, division of Pediatric Infectious Diseases, Erasmus MC-Sophia Children's Hospital, 3015GD Rotterdam, The Netherlands; a.vanrossum@erasmusmc.nl

5 Department of Pediatrics, Elisabeth-TweeSteden Hospital, 5042AD Tilburg, The Netherlands; c.obihara@etz.nl

6 Department of Pediatrics, Franciscus Gasthuis \& Vlietland, 3045PM Rotterdam, The Netherlands; g.tramper@franciscus.nl

7 Department of Medical Microbiology and Infectious Diseases, Erasmus University Medical Centre (Erasmus MC), 3015GD Rotterdam, The Netherlands; a.heikema@erasmusmc.nl (A.P.H.);

d.kreft@erasmusmc.nl (D.H.-K.); j.hays@erasmusmc.nl (J.P.H.)

8 Department of Pathology, Clinical Bioinformatics Unit, Erasmus University Medical Centre (Erasmus MC), 3015GD Rotterdam, The Netherlands; w.dekoning.1@erasmusmc.nl (W.d.K.); a.stubbs@erasmusmc.nl (A.S.)

9 Department of Pediatrics, Flevoziekenhuis, 1315RA Almere, The Netherlands; avwermeskerken@flevoziekenhuis.nl

10 Department of Pediatrics, Maastricht University Medical Center, 3584CX Maastricht, The Netherlands; gertjan.driessen@mumc.nl

11 Department of Pediatrics, Langeland Ziekenhuis, 2725NA Zoetermeer, The Netherlands; j.punt@1lz.nl

12 Department of Pediatrics, Maasstad Ziekenhuis, 3079DZ Rotterdam, The Netherlands; smitf@maasstadziekenhuis.nl

13 Department of Pediatrics, Reinier de Graaf Gasthuis, 2625AD Delft, The Netherlands; j.noordzij@rdgg.nl

* Correspondence: r.oostenbrink@erasmusmc.nl; Tel.: +31-614886807 
associated with hospitalization. Virulence groups were not significantly associated with hospitalization. Our data underlines high detection rates and co-infection of viruses in children with respiratory symptoms and confirms the predominant presence of Haemophilus-, Streptococcus-, and Moraxelladominant profiles in a symptomatic pediatric population at the ED. However, we could not assess significant associations between microbiome profiles and disease severity measures.

Keywords: lower respiratory tract infection; respiratory microbiome; virus; nanopore sequencing; 16S-rRNA gene

\section{Introduction}

Lower respiratory tract infections (LRTIs) are the leading global cause of mortality in children under 5 years old [1], with 13\% of all deaths attributed to LRTIs in 2016 [2]. In addition, LRTIs are a major cause of morbidity, as 53-62\% of hospitalizations for infectious diseases were attributed to LRTI [3]. Nonetheless, the majority of LRTI cases are mild. Therefore, the ability to adequately differentiate between mild and severe LRTI cases could be important for guiding the treatment strategy and improving LRTI outcomes.

LRTIs in children are most commonly caused by viruses [4], with respiratory syncytial virus (RSV) and influenza virus being most often implicated as causative pathogens [5] RSV and human metapneumovirus infections have been reported to pose the greatest risk for hospitalization [6]. Bacterial LRTIs in children are commonly associated with Streptococcus pneumoniae and Haemophilus influenzae infections [5].

An important role for respiratory health has been attributed to the respiratory microbiome, with reported associations between respiratory microbiome profiles and respiratory related health and disease. The respiratory microbiome also plays a role in immune system development and influences immune responses, as do local viral and bacterial interactions. [7,8]. Recent reports have demonstrated associations between the upper respiratory tract microbiome and the susceptibility and severity of LRTIs in asthmatic, health versus disease, and bronchiolitic cohorts of children [9-13]. By combining viral, respiratory microbiome, and host-related data, it has been reported that children with LRTIs can be differentiated from healthy controls [11]. Additionally, specific microbiome clusters have been associated with specific viral LRTIs and disease severity. For example, Haemophilus influenzae-enriched and Streptococcus-enriched clusters have been described to be positively associated with RSV infection and RSV-related hospitalization [13]. Yet to be discovered is whether these insights can contribute to prediction of a severe disease course within children presenting at the emergency department (ED) with symptoms of LRTIs.

In this current study, we aimed to describe the viral and microbiome spectrum and assess the potential contribution of clinical parameters, and viral infection and respiratory microbiome data, in helping to predict potential severe disease in children aged 1 month to 5 years presenting at the ED with suspected LRTIs. This study could improve our understanding of the implications of the interaction between respiratory microbiome and LRTIs and contribute to future clinical disease severity differentiation with potential implementation in clinical decision making in EDs.

\section{Materials and Methods}

\subsection{Population and Study Design}

The study population originated from a previously described cohort of 999 children aged one month to five years [14]. Briefly, children were included in the study between 1 January 2016 and 30 September 2018 by treating physicians, when they presented at the ED with fever (temperature $>38.5$ degrees Celsius) and cough or dyspnea as symptoms of a potential RTI. The eight participating centers included one tertiary (Erasmus MC Rotterdam) and seven general hospitals (Maasstad Hospital Rotterdam, Franciscus Vlietland Hospital Rotterdam, LangeLand Hospital Zoetermeer, Reinier de Graaf Hospital 
Delft, HAGA Hospital The Hague, Elisabeth-Tweesteden Hospital Tilburg, Flevo Hospital Almere) all located in the Netherlands. Exclusion criteria were defined by an increased risk of a complicated disease course, reported in more detail previously [14]. In short, an increased risk of complicated disease course included children with relevant comorbidities (immunodeficiency, congenital heart defects, chronic pulmonary disease, multiple handicaps, or prematurity), signs of complicated pneumonia, another infectious focus, antibiotic use within a week prior to the ED visit, or an amoxicillin allergy. In our study, we solely included children from whom a nasopharyngeal sample was obtained for microbiome and/or virology assessment.

\subsection{Outcomes}

The primary outcome was the rate of hospitalization as a measure of disease severity. Secondary outcomes were strategy failure and the predicted risk of having a bacterial LRTI. Strategy failure was a predefined composite outcome, based on disease course during the first 7 days after ED presentation. Predicted risk of bacterial pneumonia was computed with the Feverkidstool, a previously validated model to predict the presence of pneumonia or other serious bacterial infections in children with fever [14-16]. Three separate risk groups were defined, with cut-off points set at $<3 \%$ for low predicted risk for bacterial pneumonia, $4-10 \%$ for intermediate and $>10 \%$ for high predicted risk [14]. Detailed definitions of outcome measures are available in the online supplement.

\subsection{Data and Sample Collection}

Clinical parameters assessment and CRP (C-reactive protein) point of care (bedside) testing at ED presentation were performed by the attending nurse and physician. Extensive general and clinical data were collected on an electronic case record form. Follow-up data 7 days post initial presentation were collected through structured telephone calls or collected directly from patients and parents during the period of hospitalization. Nasopharyngeal aspirates (NFA) were obtained by the attending nurse of physician during the first ED visit, using $0.9 \% \mathrm{NaCl}, 1 \mathrm{~mL}$ in each of the nostrils. One sample was obtained per individual. Samples were stored at -80 degrees Celsius.

\subsection{Microbiome Sample Processing}

Analysis of the microbiome was performed on an extract of the NFA during a 2 day period and two negative controls (assay buffer only) were included in the extractions to check for potential contamination. 16S-rRNA sequencing was performed using a MinIon nanopore sequencer (Oxford Nanopore Technologies (ONT), Oxford, UK). The microbiome methodology, including complete DNA extraction and sequencing protocol, was verified in a parallel study (presented in a separate manuscript [17]) using defined single nasal microbiota species (obtained from the American Type Culture Collection, ATCC, Manassas, VA, USA) and is presented in detail in the online supplement. In short, DNA extraction was performed using the AGOWA mag minikit (NAP40402, LGC Genomics, Berlin, Germany), and manufacturer's instructions were followed. Nasal swab fluid with lysis buffer added to lysing matrix was compared to lysis buffer as negative controls. DNA concentrations were standardized using the Quant-iT PicoGreen dsDNA Assay kit (P7589, Invitrogen, Carlsbad, CA, USA), followed by 16S rRNA gene sequence library preparation. Basecalling of sequence reads was performed using Guppy software (version 3.1.5+781ed57, ONT, Oxford, UK). Debarcoding and classification of bacteria was performed using the EPI2ME (version 3.2.2, ONT, Oxford, UK) 16S workflow. Finally, a quality filtering step was performed was performed using a custom script with an average Q score set to 9 and identity of $85 \%$.

Sequenced samples were initially filtered for a minimum number of 1000 reads per sample, as has been standardized in other nasal microbiome studies based on Illumina sequencing [18-20]. Rarefaction curves of reads versus richness were constructed to determine the minimum number of reads required per sample to detect all bacterial species. 
Further quality filtering (read minimum, removal of rare taxa with less than $0.05 \%$ relative abundance across all samples) was performed in Calypso (University of Queensland, Brisbane, Australia) [21].

\subsection{Virology Procedures}

Samples were analyzed using real-time reverse transcriptase PCR for detection of fifteen respiratory viruses and Mycoplasma pneumoniae. The viruses tested were adenovirus (ADV), human bocavirus (HBoV), enterovirus, human metapneumovirus (hMPV), human rhinovirus (HRV), influenza A virus and influenza B virus, parainfluenza virus (PIV) types 1 through 4, respiratory syncytial virus (RSV) type A and B, and human coronavirus types NL63, OC43, and 229E. Samples were considered positive for a certain virus when cycle threshold $(\mathrm{Ct})$ values were $<40$. Subtypes of viruses were clustered together for further analyses and reporting, except for influenza A virus and influenza B virus. For the real-time reverse transcriptase PCR procedures, we followed the laboratory procedures as reported elsewhere [22,23].

Patients were categorized according to the virulence of the viruses detected in their sample, where virulence was the previously reported association of the virus with symptoms in children relative to asymptomatic controls. High virulence was assigned to those positive for hMPV, influenza type A and B, parainfluenza virus and RSV, and low virulence to children positive for bocavirus, adenovirus, coronavirus, enterovirus, and rhinovirus [24-31]. Patients with multiple viruses were assigned to the high virulence group if positive for any of the high virulence viruses. High and low virulence groups were used for further analyses.

\subsection{Analysis}

\subsubsection{Microbiome Data Analysis}

Count data were normalized using total sum scaling (TSS), where read counts are divided by the total number of reads in each sample. Relative abundance of the top 20 genera and families across all samples were calculated. Unsupervised hierarchical clustering with the Bray-Curtis distance metric was performed to identify clusters. For each identified cluster, the most abundant taxon at the genus level within the cluster was used as a classifier taxon and clusters were named accordingly. Clusters were checked for plausibility and distinctiveness by bar charts containing the top 20 genera and nonmetric multidimensional scaling plots. Richness (number of taxa) and diversity (Shannon index) are presented in bar charts and differences were tested by the Kruskal-Wallis test. Microbiome data analyses were performed using Calypso Version 8.84 (University of Queensland, Brisbane, Australia) [21].

\subsubsection{Statistical Analysis}

For the outcome measures hospitalization, strategy failure, and predicted risk of having a bacterial LRTI, we preformed univariate analyses across viruses detected in $\geq 10$ cases, across virulence groups, and across identified microbiome profiles. We used either chi-squared test or Fishers' exact test for categorical outcomes and, for numerical outcomes, one way-ANOVA for normal distributed variables or Kruskal-Wallis test as a non-parametric test. Identified differences were considered significant if $p<0.05$.

Univariate unadjusted associations between microbiome profiles, virology data and clinical parameters, and hospitalization and strategy failure were explored. Those significantly associated with the outcome measure were included in the adjusted multivariate logistic regression model. Predictors of interest were adjusted for age, gender, presence of any virus, and calculated risk of bacterial LRTI. For multivariate models, we assumed any missing data to be missing at random and handled missing data by performing multiple imputation $(\mathrm{m}=10)$ including all variables included in the analysis in the imputation model. All statistical analyses were performed using IBM SPSS version 25 (IBM Corp., Armonk, NY, USA). 


\section{Results}

\subsection{Study Population}

Of the 999 children included in the original STRAP cohort, data for microbiome profiling, virology, or both were available for 167 children. Ages ranged between 1 and 54 months, with a median age of 17 months; $62.3 \%$ were male. Children presented with a median temperature of $38.8^{\circ} \mathrm{C}$ and the most common presenting symptoms were cough $(94.5 \%)$, followed by dyspnea (79.6\%) and rales (56.2\%). Additional baseline demographic, clinical characteristics, and outcome variables are presented in Table 1 and Table S1. A microbiome profile was available for 54 children after quality filtering in Calypso [21]. The 1000 read cut-off as pre-planned and described in the method section was altered to a 10,000 read cut-off based on the results of a rarefaction curve graph. For virology analyses, 158 reliable sample results were available. A flowchart of sample inclusion and processing is shown in Figure S1.

Table 1. Baseline characteristics and management of the study population $(n=167)$.

\begin{tabular}{|c|c|c|}
\hline General Characteristics & $\mathbf{N}$ or Median & $\%$ or IQR \\
\hline Age in months * & 17 & $9-34$ \\
\hline Gender: male & 104 & 62.3 \\
\hline \multicolumn{3}{|l|}{ Season of ED visit } \\
\hline Spring & 57 & 34.1 \\
\hline Summer & 25 & 15 \\
\hline Autumn & 41 & 24.6 \\
\hline Winter & 44 & 26.3 \\
\hline \multicolumn{3}{|l|}{ Clinical presentation } \\
\hline Duration of ED stay in minutes * $(n=141)$ & 147 & $112-193$ \\
\hline Temperature at ED visit & 38.8 & 1.0 \\
\hline Duration of fever in days $(n=166)$ & 2 & $1-3$ \\
\hline Tachypnea according to APLS $(\mathrm{n}=165)$ & 145 & 87.9 \\
\hline Tachycardia according to APLS & 127 & 76 \\
\hline Saturation < $94 \%(\mathrm{n}=166)$ & 39 & 23.5 \\
\hline Capillary refill time prolonged $(n=166)$ & 17 & 10.2 \\
\hline Retractions $(\mathrm{n}=166)$ & 105 & 63.3 \\
\hline Ill appearance $(\mathrm{n}=165)$ & 70 & 42.4 \\
\hline \multicolumn{3}{|l|}{ Diagnosis } \\
\hline Pneumonia & 88 & 52.7 \\
\hline Bronchiolitis & 22 & 13.2 \\
\hline Upper RTI & 36 & 21.6 \\
\hline Subglottic laryngitis & 2 & 1.2 \\
\hline Viral induced wheeze & 18 & 10.8 \\
\hline Other & 1 & 0.6 \\
\hline \multicolumn{3}{|l|}{ Investigations } \\
\hline $\mathrm{CRP}$ in $\mathrm{mg} / \mathrm{L} *(\mathrm{n}=152)$ & 35.5 & $15-76$ \\
\hline Other lab tests performed $(n=161)$ & 41 & 25.5 \\
\hline PCR for viral diagnostics performed $(n=161)$ & 45 & 28 \\
\hline Chest $X$-ray performed & 35 & 21 \\
\hline \multicolumn{3}{|l|}{ Management and outcome } \\
\hline Hospitalization at first visit & 107 & 64.1 \\
\hline Hospitalized at any time & 111 & 66.5 \\
\hline Duration of hospitalization in days $(\mathrm{n}=164) *$ & 1 & $0-3$ \\
\hline Strategy failure $(\mathrm{n}=161)$ & 36 & 21.6 \\
\hline Antibiotics at first visit & 85 & 50.9 \\
\hline Antibiotics at any time $(\mathrm{n}=162)$ & 100 & 61.7 \\
\hline \multicolumn{3}{|l|}{ Risk groups based on Feverkidstool $^{a}(\mathrm{n}=146)$} \\
\hline Low & 30 & 20.5 \\
\hline Medium & 33 & 22.6 \\
\hline High & 83 & 56.8 \\
\hline Predicted risk of bacterial pneumonia by feverkidstool $(\mathrm{n}=146)$ * & 12.3 & $5.6-29.0$ \\
\hline Oxygen therapy received at any time & 87 & 52.1 \\
\hline
\end{tabular}

Data are presented as total $\mathrm{n}$ and percentage or median with interquartile range indicated by *. "Strategy failure" was defined by a predefined composite outcome and was based on disease course during the 7 days after ED presentation. ${ }^{\text {a }}$ Risk groups are based on the calculated predicted risk of having bacterial pneumonia using the Feverkidstool. If any data were missing, total $\mathrm{n}$ for that specific characteristic is reported. Definition of abbreviations: IQR $=$ interquartile range, $\mathrm{ED}=$ emergency department, $\mathrm{APLS}=$ advanced pediatric life support, $\mathrm{RTI}=$ respiratory tract infection, $\mathrm{CRP}=\mathrm{C}$-reactive protein, $\mathrm{PCR}=$ polymerase chain reaction. 


\subsection{Virology}

Of 158 samples, 139 (88.0\%) were positive for at least one pathogen, with $94(67.6 \%)$ samples testing positive for a single virus, 32 samples (23.0\%) for two viruses, and 13 samples $(9.4 \%)$ for more than two viruses. The most common viruses were rhinovirus $(\mathrm{n}=63,39.9 \%)$ and RSV $(\mathrm{n}=39,24.7 \%)$. The least frequently found were influenza A virus $(\mathrm{n}=5)$ and influenza $\mathrm{B}$ virus $(\mathrm{n}=2)$ (see Table 2$)$.

Table 2. Viral/mycoplasma infections and co-infections detected by PCR.

\begin{tabular}{|c|c|c|c|c|c|c|c|c|c|c|c|c|}
\hline & \multirow{2}{*}{$\begin{array}{c}\text { N positive } \\
\mathrm{N}(\%)\end{array}$} & \multirow{2}{*}{$\begin{array}{l}\text { ADV } \\
\text { Count }\end{array}$} & \multirow{2}{*}{$\begin{array}{c}\text { boca } \\
\text { Count }\end{array}$} & \multirow{2}{*}{$\begin{array}{l}\text { corona } \\
\text { Count }\end{array}$} & \multirow{2}{*}{$\begin{array}{l}\text { entero } \\
\text { Count }\end{array}$} & \multirow{2}{*}{$\begin{array}{l}\text { hMPV } \\
\text { Count }\end{array}$} & \multirow{2}{*}{$\begin{array}{l}\text { infl A } \\
\text { Count }\end{array}$} & \multirow{2}{*}{$\begin{array}{l}\text { infl B } \\
\text { Count }\end{array}$} & \multirow{2}{*}{$\begin{array}{l}\text { Myco } \\
\text { Count }\end{array}$} & \multicolumn{2}{|c|}{ parainfl rhino } & \multirow{2}{*}{$\begin{array}{l}\text { RSV } \\
\text { Count }\end{array}$} \\
\hline & & & & & & & & & & Count & Count & \\
\hline Adenovirus & $21(13.3)$ & (2) & & & & & & & & & & \\
\hline Bocavirus & $11(7.0)$ & 4 & (1) & & & & & & & & & \\
\hline Coronavirus & $10(6.3)$ & 2 & 0 & (2) & & & & & & & & \\
\hline Enterovirus & $14(8.9)$ & 2 & 0 & 1 & (6) & & & & & & & \\
\hline hMPV & $20(12.7)$ & 1 & 1 & 1 & 1 & (15) & & & & & & \\
\hline Influenza A & $5(3.2)$ & 0 & 0 & 0 & 0 & 0 & (5) & & & & & \\
\hline Influenza B & $2(1.3)$ & 0 & 0 & 0 & 1 & 0 & 0 & (1) & & & & \\
\hline Mycoplasma & $2(1.3)$ & 1 & 0 & 0 & 0 & 0 & 0 & 0 & $(0)$ & & & \\
\hline Parainfluenza & 14 (8.9) & 2 & 1 & 1 & 2 & 0 & 0 & 0 & 0 & (8) & & \\
\hline Rhinovirus & 63 (39.9) & 12 & 6 & 4 & 6 & 2 & 0 & 1 & 1 & 5 & $(33)$ & \\
\hline RSV & $39(24.7)$ & 6 & 3 & 4 & 2 & 1 & 0 & 0 & 1 & 1 & 8 & $(21)$ \\
\hline
\end{tabular}

Data are presented as the number of pathogen co-infections. The values between () indicate the number of single virus infections for that specific pathogen. Definition of abbreviations: $\mathrm{ADV}=$ adenovirus, boca $=$ bocavirus, corona $=$ coronavirus, entero $=$ enterovirus, $\mathrm{hMPV}=$ human metapneumovirus, inlf = influenza, $\mathrm{Myco}=$ Mycoplasma pneumoniae, parainfl $=$ parainfluenza virus, rhino $=$ rhinovirus, $\mathrm{RSV}=$ respiratory syncytial virus.

The composition of viral coinfections varied greatly among the viruses tested. Adenovirus, bocavirus, and coronavirus were found together with at least one other virus in $90.5 \%, 90.9 \%$, and $80 \%$ of cases, respectively. For hMPV, this was true for only $25 \%$ of cases, and influenza A virus was found only as a single virus infection (see Table 2). Children with viral co-infections were significantly younger than children with a single virus or virus negative samples (11 vs. 19.5 vs. 21 months, $p=0.002$ ).

\subsection{Association between Viral Infection and Clinical Outcome Measures}

Univariate analyses did not show significant differences between virulence groups for any of the outcome measures, although we did see a trend towards more hospitalization, strategy failure, and higher predicted risk of bacterial pneumonia for the high virulence group (Table S2).

Further separate unadjusted analyses for individual viruses showed that enterovirus was significantly associated with lower rate of hospitalization (OR (odds ratio) $0.076,95 \%$ CI $0.016-0.353, p=0.001$ ). A similar tendency was observed for adenovirus (OR $0.46,95 \%$ CI $0.182-1.161, p=0.1)$. In the multivariate analysis, this difference remained significant for enterovirus (OR $0.093,95 \%$ CI (confidence interval) $0.019-0.464, p=0.004$ ) and not for adenovirus (OR 0.388, 95\% CI 0.137-1.090, $p=0.074$ ), after adjusting for viral infection, age, gender, and Feverkidstool risk groups (see Table 3). Multivariate analyses were not performed for associations between individual viruses and strategy failure because the $p$-value for each virus was $>0.15$ in unadjusted analyses. 
Table 3. Unadjusted and adjusted associations of viruses and microbiome profiles with hospitalization.

\begin{tabular}{|c|c|c|c|c|c|c|}
\hline Pathogen & Unadjusted OR & $95 \%$ CI & sig & Adjusted OR & $95 \%$ CI & $\operatorname{sig}$ \\
\hline Adenovirus & 0.46 & $0.182-1.161$ & 0.1 & 0.388 & $0.137-1.095$ & 0.074 \\
\hline Bocavirus & 2.69 & $0.561-12.907$ & 0.216 & & & \\
\hline Enterovirus & 0.076 & $0.016-0.353$ & 0.001 & 0.078 & $0.015-0.395$ & 0.002 \\
\hline Coronavirus & 1.37 & $0.340-5.519$ & 0.685 & & & \\
\hline Rhinovirus & 1.087 & $0.559-2.114$ & 0.805 & & & \\
\hline hMPV & 1.055 & $0.395-2.818$ & 0.915 & & & \\
\hline Influenza A & 0.842 & $0.136-5.193$ & 0.853 & & & \\
\hline Parainfluenza & 0.532 & $0.177-1.602$ & 0.262 & & & \\
\hline RSV & 1.368 & $0.631-2.968$ & 0.427 & & & \\
\hline \multicolumn{7}{|l|}{ Virusgroups } \\
\hline High virulence & 1.167 & $0.411-3.315$ & 0.772 & & & \\
\hline Low virulence & 0.899 & $0.310-2.607$ & 0.845 & & & \\
\hline Negative & ref & & & & & \\
\hline PCR positive for virus & 1.038 & $0.384-2.807$ & 0.941 & & & \\
\hline \multicolumn{7}{|l|}{ Microbiome profiles } \\
\hline Haemophilus & ref & & & ref & & \\
\hline Moraxella & 1.129 & $0.287-4.441$ & 0.862 & 1.303 & $0.268-6.343$ & 0.743 \\
\hline Streptococcus & 2.118 & $0.210-21.389$ & 0.525 & 2.877 & $0.220-37.641$ & 0.420 \\
\hline
\end{tabular}

Data are presented as odds ratios with $95 \%$ confidence intervals and $p$-value as calculated by logistic regression. In the multivariate models, odds ratios were adjusted for age, gender, detection, and virus in the nasopharyngeal sample and Feverkidstool risk groups. Definition of abbreviations: $\mathrm{OR}=$ odds ratio, $\mathrm{CI}=$ confidence interval, $\mathrm{hMPV}=$ human metapneumovirus $\mathrm{RSV}=$ respiratory syncytial virus.

\subsection{Microbiome}

Relative abundance of microbiota read counts at genus and family levels are presented in Table S3. Given the explorative nature of this study, we used several analyses to define microbiome profiles. First, hierarchical clustering revealed six clusters that were obtained using the visual representation of the Bray-Curtis distance presented in the dendrogram and heatmap in Figure 1. The clusters were named as genus dominant microbiome profiles, based on the most abundant taxa on genus level within the cluster, as visualized in Figure 2. The Haemophilus-dominant profile was the most common (42.7\%), followed by the Moraxella-dominant profile (38.9\%) and the Streptococcus-dominant profile (13.0\%). Three samples were deemed outliers because they were distinctly dissimilar to any of the other samples (see Figure 1). A non-metric multi-dimensional scaling (NMDS) plot, in which the previously mentioned profiles are visualized, showed distinct multidimensional distances between the different genus-dominant profiles (Figure S2). The relative abundance of the 20 top genera in each sample is presented in Figure 2. A single genus or family dominated most samples. However, within the Moraxella-dominant profile, there was relatively high abundance of Carnobacteriaceae. At sequence read level (reads that could be assigned to species level), both richness and diversity (Shannon index) were significantly lower in Haemophilus compared to Moraxella-dominant profiles (Figure S3). 


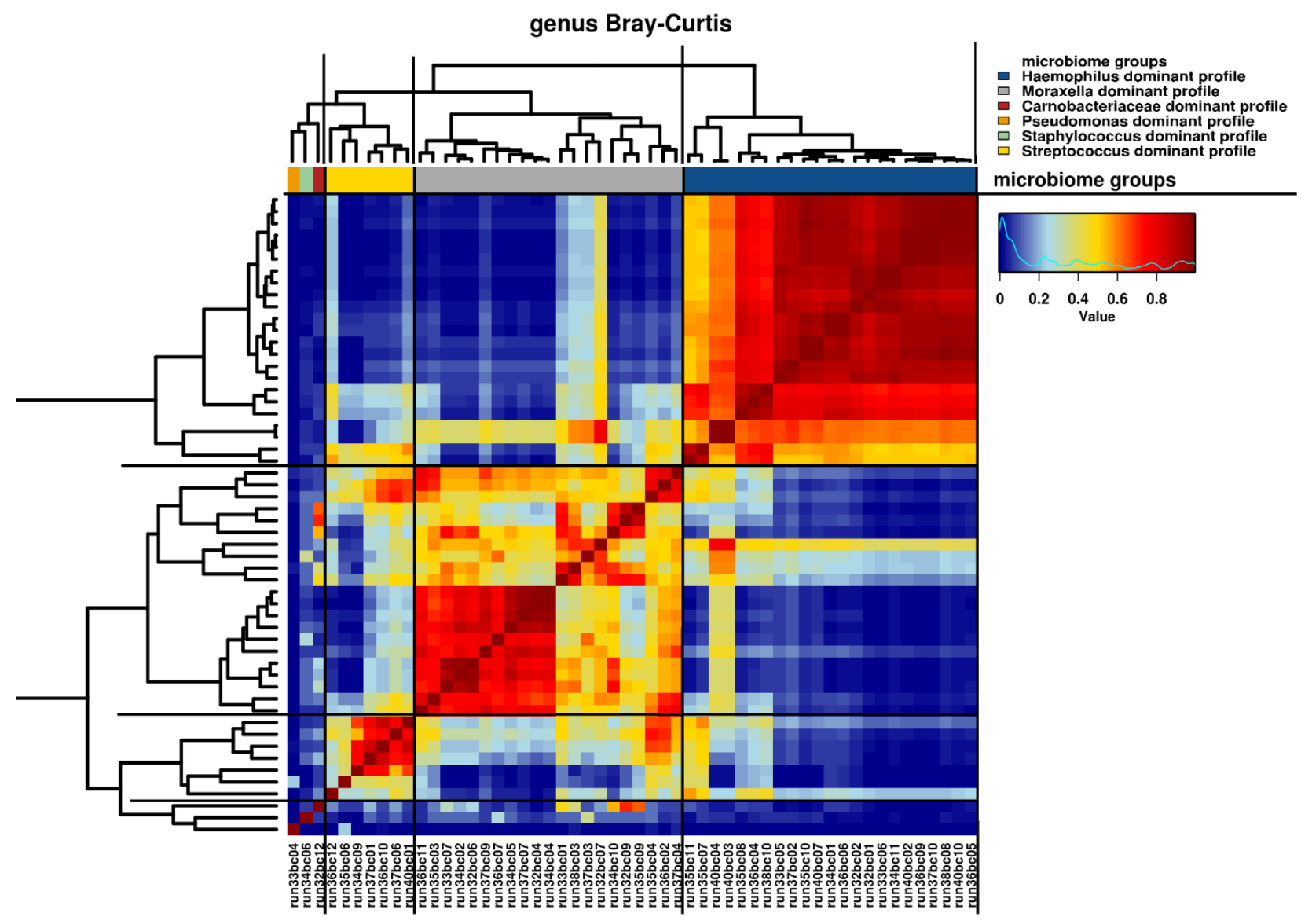

Figure 1. Hierarchical clustering of microbiome samples. Visual representation of hierarchical clustering of microbiome samples at the genus level with the Bray-Curtis distance metric. A heat map is shown, characterized by similarity values ranging from 0 to 1 . Samples are presented as run numbers. A dendrogram of the hierarchical clustering is shown with distances represented by the length of the individual branches. Genus dominant profiles are presented by colored bars, as indicated by the accompanying figure legend.

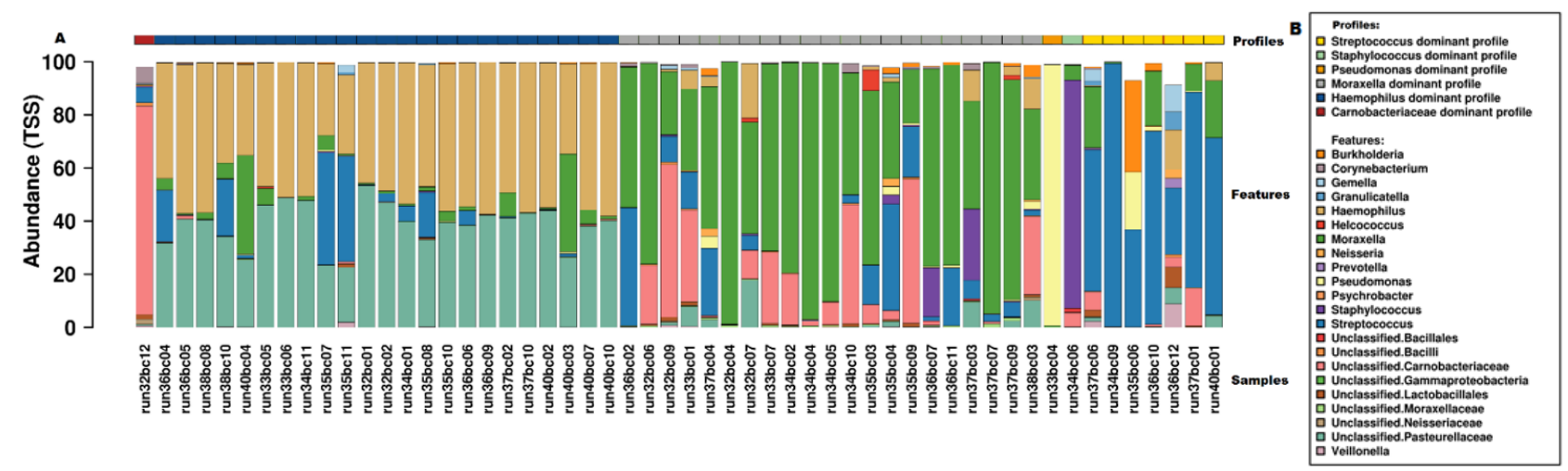

Figure 2. Microbiome profiles of samples presented as relative genus abundance. (A) Bar chart of individual samples, displaying the relative abundance on genus level for the top 20 genera. Individual samples are presented as run numbers and the corresponding microbiome profile is presented by colored bars at the top of the figure. (B) Legend for figure A.

Univariate analyses showed no significant differences between microbiome profiles for any of the clinical outcome measures (Table S4). In both unadjusted and adjusted analysis, microbiome profiles were not significantly associated with the rate of hospitalization (see Table 3). Modelling was not performed for strategy failure because there were $<10$ strategy failure cases within children with a microbiome profile present. 


\subsection{Integrating Viral, Microbiome, and Clinical Data}

Clinical descriptives for the observed microbiome profiles are presented in Table S5. Virulence groups did not differ significantly among the microbiome profiles $(p=0.697)$. Among the microbiome profiles, we observed no differences in viral presence for those viruses present in more than 10 cases (i.e., hMPV, RSV, and rhinovirus). In the absence of significant associations between microbiome profiles and severity measures we did not further explore models for hospitalization or strategy failure including clinical, virology, and microbiome predictors.

\section{Discussion}

The current publication describes a follow-up study of the STRAP trial with children aged one month to five years, who presented at the ED with fever and symptoms of RTI. We detected high numbers of viruses and viral co-infections in their respiratory samples. In multivariate analyses, enterovirus was negatively associated with hospitalization. We identified three major microbiome profiles, dominated by either Haemophilus, Moraxella, or Streptococcus. The presence of viruses was similar among the microbiome profiles. We did not observe significant associations between microbiome profiles and hospitalization, strategy failure, or predicted risk of bacterial pneumonia.

Our high viral detection rate $(88 \%)$ was comparable to that of other studies with similar populations, which reported detection rates ranging from $61 \%$ to $97 \%[6,11,25,27,29,32,33]$. Interestingly, viral detection in healthy populations was also reported to be high, with reported viral detection rates in healthy children ranging from $24.4 \%$ to $83 \%[11,25,27,29]$, questioning the causal relationship of detected viruses with respiratory symptoms. Differences in detection rate and proportion of co-infections between studies could be related to the difference in viral panels tested, with our comprehensive panel of 15 viruses contributing to our high detection rate. Additionally, differences in age distributions of study participants might have influenced detection rates [34]. In our study, children with multiple viruses were significantly younger than children with a single virus detected, in contrast with earlier studies that reported children with coinfections to be significantly older [35-37]. As in previous studies in symptomatic children (including those with severe LRTIs), RSV and rhinovirus were the most common [6,38,39]. In this study, the proportion of co-infections was high, but consistent with other studies, which reported co-infection rates ranging from $18 \%$ to $41 \%[6,32,36,40,41]$. Bocavirus and adenovirus were frequently found together with other viruses, which is confirmed by other reports $[6,32,36,40,41]$. With respect to differentiation of virus results into virulence groups, we did not see significant differences among viral virulence groups and study outcomes, although we did see a trend towards more hospitalization, strategy failure, and higher predicted risk of bacterial pneumonia for the high virulence group.

The six distinct identified microbiome profiles we identified have considerable overlap with the identified profiles in other studies. In particular, Haemophilus-, Moraxella-, and Streptococcus-dominant profiles are identified frequently [9,12,13,42,43]. However, we did not identify the Corynebacterium-dominant profile in our ED study population, in contrast to studies focusing predominantly on healthy children $[9,13,42]$. A possible explanation for this is that Corynebacterium are considered to be protective [42,44,45]. Additionally, Corynebacterium- and Staphylococcus-dominated dominated profiles have mainly been observed in younger children up to two years of age [42,44], whereas we included children up to five years of age. Also of note is that a recent publication by Heikema et al. [17], indicated possible problems with the detection of Corynebacteria using nanopore-based amplification primers compared to Illumina-based primers. Further, with respect to nanopore sequencing, we found a relatively large number of sequencing reads that could not be classified to genus level. This may have been a consequence of sequencing errors introduced by the nanopore flowcell that was used for this study (R9.2). However, nanopore sequencing is constantly evolving, with more recent evidence 
indicating a continued advancement in the ability of nanopore flowcells to identify the nasal microbiome at the genus level [17].

Previous studies have reported inconsistent associations between distinct nasopharyngeal microbiome profiles and disease severity measures [12,13,42]. Although they all identified Haemophilus-, Moraxella-, and Streptococcus-dominant profiles, among other profiles, a relative abundance of Streptococcus was associated with influenza and hospitalization [13], intensive care admission was lowest in Moraxella-dominant profiles and highest in Haemophilus-dominant profiles [12], and Streptococcus-, Haemophilus-, and Moraxelladominant profiles were significantly associated with respiratory symptoms, with the Moraxella-dominant profile associated with the severity of RSV infections [42]. We did not find significant differences between the three profiles and the disease severity outcome measures. This may result from our rather homogeneous population of symptomatic children visiting the ED, with a high hospitalization rate. However, in this ill population, we did confirm the predominant presence of the Haemophilus-, Streptococcus-, and Moraxella-dominant profiles.

\section{Strengths and Limitations}

Our study is one of the first to combine comprehensive high quality clinical data with extensive viral detection and nanopore 16S-rRNA sequencing of the respiratory microbiome.

Although we focused on LRTIs, samples were collected from the nasopharynx. Some studies indicate that the respiratory tract microbiome in general is better represented by other samples, such as tracheal aspirates, sputum, or bronchial aspirate lavages [46]. When comparing sick versus healthy children, however, it has been reported that the nasopharynx is an appropriate proxy for the lower respiratory tract microbiome in LRTIs [11]. From our observations, the use of upper respiratory tract microbiome profiling to differentiate disease severity within a cohort of children who are all presenting with symptoms of a LRTI may not yield statistically significant results. As our study was relatively small, further research is required in this area because the use of microbiome profiling of the upper respiratory tract to identify LRTIs has several advantages in terms of invasiveness and clinical and ethical viability compared to lower respiratory tract microbiome sampling.

Because collecting microbiome and virology data was a secondary aim of the original STRAP trial, the study was not designed specifically, nor powered accordingly, for the present analyses. Sample collection and analyses was therefore not implemented equally across participating hospitals and sample numbers were relatively low. This could lead to selection bias. However, our population closely resembles that of the original study population of the STRAP trial in terms of baseline characteristics, including age and gender [14]. Additionally, the data obtained regarding viral (co-)infections and microbiome profiles were similar to those of previous published reports, as described above.

With respect to virological analysis, we clustered virus subtypes into a single type (i.e., RSV, coronavirus, and parainfluenza virus) to increase the statistical validity of the results. However, this could have led to underestimation of potentially harmful subtypes of certain viruses. For RSV, equal severity of type A and B infection was assumed based on reported resemblance $[47,48]$.

Most studies on respiratory microbiome in children have used Illumina sequencing, whereas we worked with nanopore sequencing. Nanopore sequencing has several potential advantages, including long-read output, real-time analyses, and portability, but has relatively lower accuracy compared to short-read methods $[49,50]$. The long-read output, which in microbiome research could be used to sequence the full 16S-rRNA gene, could potentially lead to more accurate assembly of genome data, especially at the species level. However, the error rate in basecalling of nanopore sequencing averages up to $10 \%$ [50], meaning that microbiome profiling at the level of species, and sometimes even genus, may be problematic, as observed in this study. However, as previously mentioned, advances in nanopore sequencing technology occur regularly and newer flowcell sequencing cartridges 
appear to have reduced sequencing error rates [17]. One consequence of the potentially relatively high error rate encountered in this study was observed during rarefaction analysis, i.e., a mathematical calculation plotting the number of species against the number of samples (to generate a graph showing the number of sequencing reads against microbiota richness). Ideally, for the sample size used, a rarefaction analysis plot should show a steadily decreasing slope and plateau, with the plateau indicating that the number of samples is sufficient to obtain data from all species present within a particular microbiome. Previous publications using Illumina-based sequencing of the upper respiratory microbiome have indicated a rarefaction plateau at a minimum of approximately 1000 reads. However, from our nanopore results, a plateau was not observed, even for a sample that generated 100,000 reads. The most likely explanation for this discrepancy is the presence of errors in the nanopore sequence results that we obtained. Therefore, as a compromise, we only used samples containing a minimum of 10,000 reads for analysis and filtered out rare taxa with less than $0.05 \%$ relative abundance, maximizing the balance between accuracy and the number of remaining samples for analyses. Subsequent analysis of our microbiome data showed that the microbiome profiles obtained in this study resembled previously reported upper respiratory tract microbiome profiles obtained from symptomatic children using Illumina sequencing. Finally, in general, the range of clustering techniques used in different studies, such as partitioning around medoids (PAM) clustering $[10,12,51]$ and several hierarchal clustering techniques $[13,42]$ may impact the result comparability between microbiome-based studies.

\section{Conclusions}

This study underlines the high detection rate of viruses and viral co-infections in children with respiratory symptoms. It also highlights the presence of distinct microbiome profiles in the nasopharyngeal tract of children with respiratory symptoms at the ED, with Haemophilus-, Moraxella-, and Streptococcus-dominant profiles being the most prevalent. Although we could not assess significant associations between microbiome profiles and disease severity measures (hospitalization, strategy failure, and predicted risk of bacterial pneumonia), the predominant detection of the aforementioned profiles, compared to profiles often found in healthy children, indicates potential associations between microbiome composition and LRTIs. Further sufficiently powered research should address these relationships, and could progress to identifying and potentially implementing microbiome profiling to be used as a biomarker to identify children at risk of severe LRTIs, in combination with existing clinical severity biomarkers in EDs.

Supplementary Materials: The following are available online at https:/ / www.mdpi.com/article / 10.3390/microorganisms9071446/s1. Supplementary Materials and Methods, Table S1: Additional baseline characteristics of the study population $(n=167)$. Figure S1: Flowchart of inclusion, quality measures and sample availability, Table S2: Univariate analyses for outcome measures per viral virulence group (total $n=158$ ), Table S3: Overall presence of genera/families (relative abundance, TSS), Figure S2: NMDS plot of microbiome profiles, Figure S4: Richness and Alpha Diversity as measured by Shannon index, Table S4: Univariate analyses for outcome measures per microbiome profile (total $\mathrm{n}=51$ ), Table S5: General characteristics and virology by microbiome profile.

Author Contributions: Conceptualization, J.v.d.M., J.P.H. and R.O.; methodology, I.H., J.P.H., R.O., J.J.A.v.K., D.H.-K.; software, I.H., J.P.H., R.O., J.J.A.v.K., A.P.H.; validation, J.P.H., J.J.A.v.K., R.O.; formal analysis, I.H., J.v.d.M., J.P.H., R.O.; investigation, I.H., J.P.H., J.J.A.v.K., J.v.d.M., R.O., C.O., G.A.T.-S., A.-M.v.W., G.J.A.D., J.P., F.J.S., J.G.N., D.H.-K.; resources, all authors; data curation, I.H., J.v.d.M., J.P.H., R.O.; writing—original draft preparation, I.H., J.P.H., R.O.; writing—review and editing, I.H., J.v.d.M., J.J.A.v.K., A.v.R., C.O., G.A.T.-S., A.P.H., W.d.K., D.H.-K., J.P.H., R.O.; visualization, I.H., J.P.H., R.O.; supervision, J.P.H., J.J.A.v.K., R.O.; project administration, R.O., J.v.d.M.; funding acquisition, J.v.d.M., R.O., J.P.H., J.J.A.v.K. All authors have read and agreed to the published version of the manuscript.

Funding: This study was funded by The Netherlands Organisation for Health Research and Development (ZonMW, grant number 836041001) and Innovatiefonds Zorgverzekeraars (B14-205, dossier 2818). 
Institutional Review Board Statement: The study was conducted according to the guidelines of the Declaration of Helsinki, and approved by the Ethics Committee of the Erasmus MC (MEC-2014-332, 04-03-2015).

Informed Consent Statement: Informed consent was obtained from all subjects involved in the study.

Data Availability Statement: Individual participant data, virology data and microbiota data that underlie the reported results will be available after de-identification at time of article publication, ending 10 years following article publication. Data will be shared with investigators who provide a methodologically sound proposal, or for individual participant data meta-analysis. Data are deposited in the repository of Data Archiving and Networked Services (DANS, doi: 10.17026/dans27a-fj4k). Proposals should be directed to info@dans.knaw.nl; to gain access, data requestors will need to sign a data access agreement.

Acknowledgments: We gratefully acknowledge all doctors, nurses, research nurses and medical students that contributed to the data collection in the participating hospitals. We thank all the included patients and their parents for their willingness to participate in the trial. We thank D. Bogaert, University of Edinburgh for her advice during the project.

Conflicts of Interest: The authors declare no conflict of interest. The funders had no role in the design of the study; in the collection, analyses, or interpretation of data; in the writing of the manuscript, or in the decision to publish the results.

\section{References}

1. Collaborators GBDCoD. Global, regional, and national age-sex specific mortality for 264 causes of death, 1980-2016: A systematic analysis for the Global Burden of Disease Study 2016. Lancet 2017, 390, 1151-1210. [CrossRef]

2. Collaborators GBDLRI. Estimates of the global, regional, and national morbidity, mortality, and aetiologies of lower respiratory infections in 195 countries, 1990-2016: A systematic analysis for the Global Burden of Disease Study 2016. Lancet Infect. Dis. 2018, 18, 1191-1210. [CrossRef]

3. Goto, T.; Tsugawa, Y.; Mansbach, J.M.; Camargo, C.A., Jr.; Hasegawa, K. Trends in Infectious Disease Hospitalizations in US Children, 2000 to 2012. Pediatr. Infect. Dis. J. 2016, 35, e158-e163. [CrossRef]

4. Tramper-Stranders, G.A. Childhood community-acquired pneumonia: A review of etiology- and antimicrobial treatment studies. Paediatr. Respir. Rev. 2018, 26, 41-48. [CrossRef] [PubMed]

5. $\quad$ Rudan, I.; O’Brien, K.L.; Nair, H.; Liu, L.; Theodoratou, E.; Qazi, S.; Luksic, I.; Fischer Walker, C.L.; Black, R.E.; Campbell, H.; et al. Epidemiology and etiology of childhood pneumonia in 2010: Estimates of incidence, severe morbidity, mortality, underlying risk factors and causative pathogens for 192 countries. J. Glob. Health 2013, 3, 010401.

6. Ortiz-Hernández, A.A.; Nishimura, K.K.; Noyola, D.E.; Moreno-Espinosa, S.; Gamiño, A.; Galindo-Fraga, A.; Valdéz Vázquez, R.; Magaña Aquino, M.; Ramirez-Venegas, A.; Valdés Salgado, R.; et al. Differential risk of hospitalization among single virus infections causing influenza-like illnesses. Influenza Other Respir. Viruses 2019, 13, 36-43. [CrossRef] [PubMed]

7. Man, W.H.; de Steenhuijsen Piters, W.A.; Bogaert, D. The microbiota of the respiratory tract: Gatekeeper to respiratory health Nat. Rev. Microbiol. 2017, 15, 259-270. [CrossRef] [PubMed]

8. Neu, U.; Mainou, B.A. Virus interactions with bacteria: Partners in the infectious dance. PLoS Pathog. 2020, 16, e1008234. [CrossRef] [PubMed]

9. McCauley, K.; Durack, J.; Valladares, R.; Fadrosh, D.W.; Lin, D.L.; Calatroni, A.; LeBeau, P.K.; Tran, H.T.; Fujimura, K.E.; LaMere, B.; et al. Distinct nasal airway bacterial microbiotas differentially relate to exacerbation in pediatric patients with asthma. $J$. Allergy Clin. Immunol. 2019, 144, 1187-1197. [CrossRef]

10. Hasegawa, K.; Linnemann, R.W.; Mansbach, J.M.; Ajami, N.J.; Espinola, J.A.; Petrosino, J.F.; Piedra, P.A.; Stevenson, M.D.; Sullivan, A.F.; Thompson, A.D.; et al. Nasal Airway Microbiota Profile and Severe Bronchiolitis in Infants: A Case-control Study. Pediatr. Infect. Dis. J. 2017, 36, 1044-1051. [CrossRef] [PubMed]

11. Man, W.H.; van Houten, M.A.; Mérelle, M.E.; Vlieger, A.M.; Chu, M.; Jansen, N.J.G.; Sanders, E.A.M.; Bogaert, D. Bacterial and viral respiratory tract microbiota and host characteristics in children with lower respiratory tract infections: A matched case-control study. Lancet Respir. Med. 2019, 7, 417-426. [CrossRef]

12. Hasegawa, K.; Mansbach, J.M.; Ajami, N.J.; Espinola, J.A.; Henke, D.M.; Petrosino, J.F.; Piedra, P.A.; Shaw, C.A.; Sullivan, A.F.; Camargo, C.A., Jr.; et al. Association of nasopharyngeal microbiota profiles with bronchiolitis severity in infants hospitalised for bronchiolitis. Eur. Respir. J. 2016, 48, 1329-1339. [CrossRef]

13. de Steenhuijsen Piters, W.A.; Heinonen, S.; Hasrat, R.; Bunsow, E.; Smith, B.; Suarez-Arrabal, M.C.; Chaussabel, D.; Cohen, D.M.; Sanders, E.A.; Ramilo, O.; et al. Nasopharyngeal Microbiota, Host Transcriptome, and Disease Severity in Children with Respiratory Syncytial Virus Infection. Am. J. Respir. Crit. Care Med. 2016, 194, 1104-1115. [CrossRef] [PubMed]

14. van de Maat, J.S.; Peeters, D.; Nieboer, D.; van Wermeskerken, A.M.; Smit, F.J.; Noordzij, J.G.; Tramper-Stranders, G.; Driessen, G.J.A.; Obihara, C.C.; Punt, J.; et al. Evaluation of a clinical decision rule to guide antibiotic prescription in children with suspected 
lower respiratory tract infection in The Netherlands: A stepped-wedge cluster randomised trial. PLoS Med. 2020, 17, e1003034. [CrossRef] [PubMed]

15. Nijman, R.G.; Vergouwe, Y.; Moll, H.A.; Smit, F.J.; Weerkamp, F.; Steyerberg, E.W.; van der Lei, J.; de Rijke, Y.B.; Oostenbrink, R. Validation of the Feverkidstool and procalcitonin for detecting serious bacterial infections in febrile children. Pediatr. Res. 2018, 83, 466-476. [CrossRef] [PubMed]

16. Nijman, R.G.; Vergouwe, Y.; Thompson, M.; van Veen, M.; van Meurs, A.H.; van der Lei, J.; Steyerberg, E.W.; Moll, H.A.; Oostenbrink, R. Clinical prediction model to aid emergency doctors managing febrile children at risk of serious bacterial infections: Diagnostic study. BMJ 2013, 346, f1706. [CrossRef] [PubMed]

17. Heikema, A.P.; Horst-Kreft, D.; Boers, S.A.; Jansen, R.; Hiltemann, S.D.; de Koning, W.; Kraaij, R.; de Ridder, M.A.J.; van Houten, C.B.; Bont, L.J.; et al. Comparison of Illumina versus Nanopore $16 \mathrm{~S}$ rRNA Gene Sequencing of the Human Nasal Microbiota. Genes 2020, 11, 1105. [CrossRef] [PubMed]

18. De Boeck, I.; Wittouck, S.; Wuyts, S.; Oerlemans, E.F.M.; van den Broek, M.F.L.; Vandenheuvel, D.; Vanderveken, O.; Lebeer, S. Comparing the Healthy Nose and Nasopharynx Microbiota Reveals Continuity as Well as Niche-Specificity. Front. Microbiol. 2017, 8, 2372. [CrossRef]

19. Gan, W.; Yang, F.; Tang, Y.; Zhou, D.; Qing, D.; Hu, J.; Liu, S.; Liu, F.; Meng, J. The difference in nasal bacterial microbiome diversity between chronic rhinosinusitis patients with polyps and a control population. Int. Forum Allergy Rhinol. 2019, 9, 582-592. [CrossRef] [PubMed]

20. Depner, M.; Ege, M.J.; Cox, M.J.; Dwyer, S.; Walker, A.W.; Birzele, L.T.; Genuneit, J.; Horak, E.; Braun-Fahrlander, C.; Danielewicz, H.; et al. Bacterial microbiota of the upper respiratory tract and childhood asthma. J. Allergy Clin. Immunol. 2017, 139, 826-834.e13. [CrossRef]

21. Zakrzewski, M.; Proietti, C.; Ellis, J.J.; Hasan, S.; Brion, M.J.; Berger, B.; Krause, L. Calypso: A user-friendly web-server for mining and visualizing microbiome-environment interactions. Bioinformatics 2017, 33, 782-783. [CrossRef] [PubMed]

22. Hoek, R.A.; Paats, M.S.; Pas, S.D.; Bakker, M.; Hoogsteden, H.C.; Boucher, C.A.; van der Eerden, M.M. Incidence of viral respiratory pathogens causing exacerbations in adult cystic fibrosis patients. Scand. J. Infect. Dis. 2013, 45, 65-69. [CrossRef] [PubMed]

23. Moesker, F.M.; van Kampen, J.J.A.; Aron, G.; Schutten, M.; van de Vijver, D.; Koopmans, M.P.G.; Osterhaus, A.D.M.E.; Fraaij, P.L.A. Diagnostic performance of influenza viruses and RSV rapid antigen detection tests in children in tertiary care. J. Clin. Virol. 2016, 79, 12-17. [CrossRef]

24. Spuesens, E.B.; Fraaij, P.L.; Visser, E.G.; Hoogenboezem, T.; Hop, W.C.; van Adrichem, L.N.; Weber, F.; Moll, H.A.; Broekman, B.; Berger, M.Y.; et al. Carriage of Mycoplasma pneumoniae in the upper respiratory tract of symptomatic and asymptomatic children: An observational study. PLoS Med. 2013, 10, e1001444. [CrossRef]

25. Jansen, R.R.; Wieringa, J.; Koekkoek, S.M.; Visser, C.E.; Pajkrt, D.; Molenkamp, R.; de Jong, M.D.; Schinkel, J. Frequent detection of respiratory viruses without symptoms: Toward defining clinically relevant cutoff values. J. Clin. Microbiol. 2011, 49, 2631-2636. [CrossRef]

26. Chonmaitree, T.; Alvarez-Fernandez, P.; Jennings, K.; Trujillo, R.; Marom, T.; Loeffelholz, M.J.; Miller, A.L.; McCormick, D.P.; Patel, J.A.; Pyles, R.B. Symptomatic and asymptomatic respiratory viral infections in the first year of life: Association with acute otitis media development. Clin. Infect. Dis. 2015, 60, 1-9. [CrossRef]

27. Self, W.H.; Williams, D.J.; Zhu, Y.; Ampofo, K.; Pavia, A.T.; Chappell, J.D.; Hymas, W.C.; Stockmann, C.; Bramley, A.M.; Schneider, E.; et al. Respiratory Viral Detection in Children and Adults: Comparing Asymptomatic Controls and Patients with Community-Acquired Pneumonia. J. Infect. Dis. 2016, 213, 584-591. [CrossRef]

28. Spichak, T.V.; Yatsyshina, S.; Katosova, L.K.; Kim, S.S.; Korppi, M.O. Is the role of rhinoviruses as causative agents of pediatric community-acquired pneumonia over-estimated? Eur. J. Pediatr. 2016, 175, 1951-1958. [CrossRef]

29. Singleton, R.J.; Bulkow, L.R.; Miernyk, K.; DeByle, C.; Pruitt, L.; Hummel, K.B.; Bruden, D.; Englund, J.A.; Anderson, L.J.; Lucher, L.; et al. Viral respiratory infections in hospitalized and community control children in Alaska. J. Med. Virol. 2010, 82, 1282-1290. [CrossRef]

30. Sarna, M.; Lambert, S.B.; Sloots, T.P.; Whiley, D.M.; Alsaleh, A.; Mhango, L.; Bialasiewicz, S.; Wang, D.; Nissen, M.D.; Grimwood, K.; et al. Viruses causing lower respiratory symptoms in young children: Findings from the ORChID birth cohort. Thorax 2018, 73, 969-979. [CrossRef]

31. Shi, T.; McLean, K.; Campbell, H.; Nair, H. Aetiological role of common respiratory viruses in acute lower respiratory infections in children under five years: A systematic review and meta-analysis. J. Glob. Health 2015, 5, 010408. [CrossRef]

32. Brand, H.K.; de Groot, R.; Galama, J.M.; Brouwer, M.L.; Teuwen, K.; Hermans, P.W.; Melchers, W.J.; Warris, A. Infection with multiple viruses is not associated with increased disease severity in children with bronchiolitis. Pediatr. Pulmonol. 2012, 47, 393-400. [CrossRef] [PubMed]

33. Toizumi, M.; Suzuki, M.; Nguyen, H.A.T.; Le, M.N.; Ariyoshi, K.; Moriuchi, H.; Hashizume, M.; Dang, D.A.; Yoshida, L.M. Viral Acute Respiratory Illnesses in Young Infants Increase the Risk of Respiratory Readmission. Pediatr. Infect. Dis. J. 2018, 37, 1217-1222. [CrossRef] [PubMed]

34. Weigl, J.A.; Puppe, W.; Grondahl, B.; Schmitt, H.J. Epidemiological investigation of nine respiratory pathogens in hospitalized children in Germany using multiplex reverse-transcriptase polymerase chain reaction. Eur. J. Clin. Microbiol. Infect. Dis. 2000, 19, 336-343. [CrossRef] [PubMed] 
35. Peng, D.; Zhao, D.; Liu, J.; Wang, X.; Yang, K.; Xicheng, H.; Yang, L.; Wang, F. Multipathogen infections in hospitalized children with acute respiratory infections. Virol. J. 2009, 6, 155. [CrossRef] [PubMed]

36. Wishaupt, J.O.; van der Ploeg, T.; de Groot, R.; Versteegh, F.G.; Hartwig, N.G. Single- and multiple viral respiratory infections in children: Disease and management cannot be related to a specific pathogen. BMC Infect. Dis. 2017, 17, 62. [CrossRef] [PubMed]

37. Rotzen-Ostlund, M.; Eriksson, M.; Tiveljung Lindell, A.; Allander, T.; Zweygberg Wirgart, B.; Grillner, L. Children with multiple viral respiratory infections are older than those with single viruses. Acta Paediatr. 2014, 103, 100-104. [CrossRef]

38. Jennings, L.C.; Anderson, T.P.; Werno, A.M.; Beynon, K.A.; Murdoch, D.R. Viral etiology of acute respiratory tract infections in children presenting to hospital: Role of polymerase chain reaction and demonstration of multiple infections. Pediatr. Infect. Dis. J. 2004, 23, 1003-1007. [CrossRef]

39. Moesker, F.M.; van Kampen, J.J.; van Rossum, A.M.; de Hoog, M.; Koopmans, M.P.; Osterhaus, A.D.; Fraaij, P.L. Viruses as Sole Causative Agents of Severe Acute Respiratory Tract Infections in Children. PLoS ONE 2016, 11, e0150776. [CrossRef]

40. Rhedin, S.; Lindstrand, A.; Hjelmgren, A.; Ryd-Rinder, M.; Ohrmalm, L.; Tolfvenstam, T.; Ortqvist, A.; Rotzen-Ostlund, M.; Zweygberg-Wirgart, B.; Henriques-Normark, B.; et al. Respiratory viruses associated with community-acquired pneumonia in children: Matched case-control study. Thorax 2015, 70, 847-853. [CrossRef]

41. Martin, E.T.; Kuypers, J.; Wald, A.; Englund, J.A. Multiple versus single virus respiratory infections: Viral load and clinical disease severity in hospitalized children. Influenza Other Respir. Viruses 2012, 6, 71-77. [CrossRef] [PubMed]

42. Teo, S.M.; Mok, D.; Pham, K.; Kusel, M.; Serralha, M.; Troy, N.; Holt, B.J.; Hales, B.J.; Walker, M.L.; Hollams, E.; et al. The infant nasopharyngeal microbiome impacts severity of lower respiratory infection and risk of asthma development. Cell Host Microbe 2015, 17, 704-715. [CrossRef]

43. Stewart, C.J.; Mansbach, J.M.; Wong, M.C.; Ajami, N.J.; Petrosino, J.F.; Camargo, C.A., Jr.; Hasegawa, K. Associations of Nasopharyngeal Metabolome and Microbiome with Severity among Infants with Bronchiolitis. A Multiomic Analysis. Am. J. Respir. Crit. Care Med. 2017, 196, 882-891. [CrossRef]

44. Biesbroek, G.; Tsivtsivadze, E.; Sanders, E.A.; Montijn, R.; Veenhoven, R.H.; Keijser, B.J.; Bogaert, D. Early respiratory microbiota composition determines bacterial succession patterns and respiratory health in children. Am. J. Respir. Crit. Care Med. 2014, 190, 1283-1292. [CrossRef]

45. Laufer, A.S.; Metlay, J.P.; Gent, J.F.; Fennie, K.P.; Kong, Y.; Pettigrew, M.M. Microbial communities of the upper respiratory tract and otitis media in children. mBio 2011, 2, e00245-10. [CrossRef]

46. Watson, R.L.; de Koff, E.M.; Bogaert, D. Characterising the respiratory microbiome. Eur. Respir. J. 2019, 53, 1801711. [CrossRef] [PubMed]

47. Fodha, I.; Vabret, A.; Ghedira, L.; Seboui, H.; Chouchane, S.; Dewar, J.; Gueddiche, N.; Trabelsi, A.; Boujaafar, N.; Freymuth, F. Respiratory syncytial virus infections in hospitalized infants: Association between viral load, virus subgroup, and disease severity. J. Med. Virol. 2007, 79, 1951-1958. [CrossRef] [PubMed]

48. Laham, F.R.; Mansbach, J.M.; Piedra, P.A.; Hasegawa, K.; Sullivan, A.F.; Espinola, J.A.; Camargo, C.A., Jr. Clinical Profiles of Respiratory Syncytial Virus Subtypes A and B Among Children Hospitalized with Bronchiolitis. Pediatr. Infect. Dis. J. 2017, 36, 808-810. [CrossRef]

49. Shin, J.; Lee, S.; Go, M.J.; Lee, S.Y.; Kim, S.C.; Lee, C.H.; Cho, B.K. Analysis of the mouse gut microbiome using full-length 16S rRNA amplicon sequencing. Sci. Rep. 2016, 6, 29681. [CrossRef]

50. Rang, F.J.; Kloosterman, W.P.; de Ridder, J. From squiggle to basepair: Computational approaches for improving nanopore sequencing read accuracy. Genome Biol. 2018, 19, 90. [CrossRef]

51. Toivonen, L.; Hasegawa, K.; Waris, M.; Ajami, N.J.; Petrosino, J.F.; Camargo, C.A., Jr.; Peltola, V. Early nasal microbiota and acute respiratory infections during the first years of life. Thorax 2019, 74, 592-599. [CrossRef] [PubMed] 